(C) 2022, The Authors. Published by Elsevier Inc. and Fass Inc. on behalf of the American Dairy Science Association ${ }^{\circledR}$. This is an open access article under the CC BY license (http://creativecommons.org/licenses/by/4.0/).

\title{
Streptococcus dysgalactiae ssp. dysgalactiae in Norwegian bovine dairy herds: Risk factors, sources, and genomic diversity
}

\author{
M. Smistad, ${ }^{1,2 *} \odot$ H. Kaspersen, ${ }^{1} \odot$ F. V. Franklin-Alming,,${ }^{1}$ C. Wolff, ${ }^{1} \odot$ L. Sølverød, ${ }^{2}$ D. Porcellato, ${ }^{3}{ }^{\oplus}$ \\ E. Trettenes, ${ }^{1}$ and H. J. Jørgensen ${ }^{1}$ (i) \\ ${ }^{1}$ Norwegian Veterinary Institute, 1431 Ås, Norway \\ ${ }^{2}$ TINE Mastitis Laboratory, N-6415 Molde, Norway \\ ${ }^{3}$ Faculty of Chemistry, Biotechnology and Food Science, Norwegian University of Life Sciences, N-1432 Ås, Norway
}

\begin{abstract}
Despite the importance of Streptococcus dysgalactiae ssp. dysgalactiae (SDSD) as an udder pathogen, the reservoir and epidemiological characteristics of this bacterium are largely unexplored. The aims of this study were to investigate risk factors for SDSD intramammary infections (SDSD-IMI) in Norwegian bovine dairy herds, identify sources of SDSD on animals and in the environment, and elucidate the genetic diversity of SDSD isolates. Data from herd recordings and a questionnaire were used to investigate herdlevel risk factors for SDSD-IMI in 359 freestall dairy herds. Seven herds with a suspected high prevalence of SDSD-IMI were visited to sample extramammary sources (e.g., skin, wounds, mucous membranes, and freestall environment). Bacterial isolates were wholegenome sequenced to investigate the distribution of SDSD genotypes within herds and to assess the phylogenetic relationship between SDSD isolates from 27 herds across Norway. Risk factors for high incidence of SDSD-IMI in freestall dairy herds were related to housing, including closed flooring in alleys and rubber mats in cubicle bases. Parlor milking was also a risk factor compared with automatic milking systems. From herd visits, a considerable proportion of extramammary samples were SDSD positive, particularly from wounds and skin of the animals and the cubicle bases. Samples from mucous surfaces (nostrils, rectum, and vagina) and water troughs were least frequently positive. Eight multilocus sequence types (ST) were identified among the sequenced isolates from 27 herds, and phylogenetic analyses revealed 8 clades corresponding to ST. No significant association was identified between sampling site (milk, body sites, and environment) and ST. In 4 of 6 herds from which 5 or more isolates were
\end{abstract}

Received October 22, 2021.

Accepted January 5, 2022.

*Corresponding author: marit.smistad@tine.no available, one ST dominated and was found in milk and extramammary samples. One ST (ST453) was found in 15 of 27 herds, which implies that this is a widely distributed and possibly a bovine-adapted strain. Findings in this study suggest that SDSD is a cow-adapted opportunist with potential for contagious transmission, and that the freestall environment is likely to play a role in transmission between cows.

Key words: intramammary infection, multilocus sequence type, mastitis, whole-genome sequencing

\section{INTRODUCTION}

Streptococcus dysgalactiae ssp. dysgalactiae (SDSD) is an important pathogen of the bovine udder, with the ability to cause severe clinical mastitis, prolonged elevated SCC, and decreased milk yield (Whist et al., 2007; Ericsson Unnerstad et al., 2009; Heikkilä et al., 2018). It is among the major causes of IMI of dairy cows in Norway and several other countries (TINE, 2020; Duse et al., 2021; Kabelitz et al., 2021), and has negative impacts on animal welfare, economy, and antimicrobial use. According to TINE Mastitis Laboratory in Norway, the proportion of milk samples positive for SDSD increased from $6 \%$ to $13 \%$ between 2002 and 2020 (TINE, 2020).

Relatively few studies have investigated risk factors for infection of the bovine udder with SDSD (SDSDIMI), or the transmission pathways and pathogenicity of this bacterium. A study from 1999 reported that an elevated incidence rate of clinical mastitis caused by SDSD in Dutch dairy herds was associated with nutrition and milking technique (Barkema et al., 1999). A more recent Finnish study showed that cows milked in a milking parlor had a higher risk of SDSD-IMI compared with cows milked in other systems (Taponen et al., 2017). The authors of that study hypothesized that this could be related to the age and design of freestall barns with parlor milking.

Questions remain whether SDSD is a contagious udder pathogen, whether it is more of an opportunist, 
and whether strains differ in their pathogenic potential. Although SDSD is a common cause of subclinical mastitis in Norwegian dairy cows (TINE, 2020), it can also cause severe clinical mastitis and, in our experience, can also affect herds in an outbreak-like fashion, with many IMI cases in a short period.

Using various strain-typing schemes, previous studies have suggested that the strain diversity, and hence also the epidemiology of SDSD, may differ between farms. For example, several different SDSD genotypes can be found in individual dairy farms, as might be expected for opportunistic bacteria (Gillespie et al., 1998; Wang et al., 1999; Lundberg et al., 2016; Wente and Krömker, 2020). However, the same studies also showed that in some herds one strain dominated and could be found in milk from multiple cows. This suggests transmission between cows and that some strains might be more contagious than others.

Several studies have shown that new SDSD-IMI may establish during the dry period and in heifers before calving, even if they are not housed with lactating animals (Todhunter et al., 1995; Persson Waller et al., 2009). This suggests that sources other than infected udders may play a role in transmission, but few studies have systematically investigated sources of SDSD in dairy farms. Streptococcus dysgalactiae ssp. dysgalactiae has been detected in tonsils of cattle and calves (Daleel and Frost, 1967; Cruz Colque et al., 1993), and, more recently, Lundberg detected it on the skin of one cow and a wound of another cow (Lundberg, 2015). Streptococcus dysgalactiae ssp. dysgalactiae has also been found on flies caught in the farm environment (Bramley et al., 1985; Chirico et al., 1997). To our knowledge, with the exception of SDSD-positive flies, no studies have yet detected SDSD in the environment of dairy herds.

The genetic diversity of SDSD from Norwegian dairy herds is unknown, and we do not know whether certain bacterial genotypes are more often associated with mastitis than others. Previous studies investigating between-herd diversity of SDSD have used gel-based genotyping methods (Baseggio et al., 1997; Wang et al., 1999; Lundberg et al., 2014; Wente and Krömker, 2020) or biotyping (Aarestrup and Jensen, 1996). New studies using whole-genome sequencing (WGS), which has higher resolution, to investigate within-herd and between-herd diversity of SDSD are warranted.

Prevention of SDSD-IMI in dairy herds should include avoiding risk factors and reducing exposure of the cow and udder to the bacterium. The aims of the present study were to identify herd-level risk factors for SDSD-IMI in Norwegian bovine dairy herds and to investigate extramammary sources of SDSD and their possible role in transmission between cows.

\section{MATERIALS AND METHODS}

\section{Norwegian Bovine Dairy Herds and Herd Recording System}

In 2020, the Norwegian bovine dairy industry consisted of 7,214 herds with an average size of 29 lactating cows, and an annual milk yield of $8,204 \mathrm{~kg}$ (Statistics Norway, 2020). A total of $65 \%$ of Norwegian dairy cows were housed in freestalls, and $57 \%$ of the milk was produced in automatic milking systems (AMS). Norwegian Red was the main breed, accounting for $92 \%$ of the national herd (TINE, 2020).

The Norwegian Herd Recording System (NHRS) records production parameters such as milk recordings, farm characteristics, disease treatments, and bacteriological results of milk samples submitted to the accredited TINE Mastitis Laboratory. A total of 98\% of Norwegian dairy farmers report to the NHRS (TINE, 2020).

\section{Study Design, Data Collection, and Statistical Analyses}

This study consisted of 2 parts. The first was a retrospective case-control study of herd-level risk factors for SDSD-IMI, based on data from NHRS and a farmer survey. The second was a cross-sectional field study with bacteriological investigations in 7 freestall dairy herds to identify sources of SDSD, which included WGS of SDSD for genomic characterization and phylogenetic investigation.

\section{Risk Factor Study}

Herd-level data were retrieved from the approximately 8,000 farms reporting to NHRS for the period of January 1, 2017, to December 30, 2018, including barn type, milking system, herd size, and results from bacteriological testing of milk samples. Herds with $\geq 20$ cows and submitting milk samples from at least $5 \%$ of the cows $(\mathrm{n}=1,997)$ were defined as eligible for inclusion as cases or controls, and, from these, herds were selected as follows. First, the incidence proportion of SDSD-IMI (number of cows with SDSD-IMI per annual average total number of cows) was calculated for each herd. Following preliminary investigation of data, it was decided that herds with incidence proportions above the 90th percentile and below the 10th percentile - that is, the herds with the $10 \%$ highest or $10 \%$ lowest proportions of SDSD-IMI - should be defined as case herds and control herds, respectively. The 90th percentile cut-off for "high proportion of cows with SDSD-IMI" (cases) was 
$7 \%$ (range $7-27 \%$ ), and the 10th percentile cut-off for "low proportion of cows with SDSD-IMI" (controls) was $0 \%$. All the farms with no detected cases of SDSD-IMI $(39 \%)$ were therefore defined as controls. In total, 984 farms (215 cases and 769 controls) fulfilled the inclusion criteria, and the survey was sent to these farmers.

For the farmer survey, a questionnaire about herd management was created in Norwegian (English translation is available upon request). The questionnaire was designed in Questback (www.questback.com), included 46 questions, and took about $10 \mathrm{~min}$ to complete. Most questions were closed, or semi-closed, and, where relevant, space was provided for comments. Before distribution, 3 dairy farmers tested the questionnaire to ensure clarity, and some minor adjustments were made following feedback. The survey was distributed by e-mail in November 2019 to all selected farmers. One e-mail reminder was sent after 3 wk. To encourage participation, one participant was randomly selected to win a tablet (iPad 6th gen., 2018, Apple Inc.). Questionnaire data were merged with the data retrieved from NHRS for further analyses. All data were collected and stored according to the EU General Data Protection Regulation.

Data for the risk factor study were described and summarized. Because $79 \%$ of the herds with a high proportion of SDSD-IMI were freestall herds, and many of the investigated risk factors (e.g., type of flooring, introduction of heifers to the milking department) were relevant only for freestalls, the risk factor study included freestalls only.

For the risk factor analysis, unconditional associations between the dependent variable (case or control herd) and each of the potential risk factors were screened using $\chi^{2}$ or Fisher's exact tests (categorical variables) or univariable logistic regression (continuous). Variables with a $P$-value $\leq 0.2$ (Supplemental Table S1, https:/ /doi.org/10.5281/zenodo.5959175) were evaluated in a multivariable logistic regression model. Tabulation was used to assess collinearity between the predictors. For correlated variables, the one with the suspected highest biological relevance was selected. The model was built using manual backward elimination. Variables were removed from the model based on a likelihood ratio test at each step, with $P<0.05$ as criterion for retention. Herd size was forced into the model as a potential confounder to milking system. Biologically plausible interaction terms between the main effects were tested one at a time in the final model. The fit of the model was evaluated with the Hosmer-Lemeshow goodness of fit test (Dohoo et al., 2014). Data were analyzed in Stata (Release 14.2, Stata Corp. LLC, 2015).

\section{Visits and Sampling in 7 Bovine Dairy Herds}

Based on the results of the risk factor study, 7 freestall dairy farms located in Eastern Norway, designated A through $G$, were selected for a visit between January and March 2020. Two of the farms (A and G) were selected because they had SDSD as the main finding in milk samples the year before the visit (2019), as seen in NHRS data. The remaining 5 herds were selected based on increasing bulk milk SCC the month before the visit but with unknown prevalence of SDSD-IMI. The herds differed with respect to milking system (AMS or parlor), AMS type (DeLaval and Lely), and flooring in alleys (slatted or closed).

A sampling protocol was developed for farm visits. Because culturing of SDSD from contaminated samples is laborious (Smistad et al., 2021), the upper limit of samples for each visit was 90 . Samples were collected from milk, animal body sites, the stall environment, and milking machine. Quarter milk samples were obtained by standard aseptic technique from the 10 cows with the highest SCC at the last milk recording before the visit.

Swabs with $1 \mathrm{~mL}$ of liquid Amies (E-swabs or M 40 Transystem, Copan Diagnostics Inc.) were used for sampling of body sites and environment. Swab samples from the same 10 cows, selected as previously described, were obtained from the skin between the udder and the thigh, hereafter termed "udder skin," and teat skin. Any visible hock lesions or other wounds were also sampled from the same 10 animals, and if fewer than 10 of these cows had wounds, wound swabs from other cows in the milking department were collected so that at least 10 wound samples were obtained from each herd. In addition, the vagina, nose, and rectum were sampled from the first 5 of the 10 cows. Vaginal and rectal swabs were collected by inserting the swab 3 to $5 \mathrm{~cm}$ into the vagina, nostril, or rectum, and gently rolling the swab over the mucosa.

Swab samples from cubicles were obtained from 8 different sites of the freestall in each farm. From the milking machine, swabs were collected from the liners, milk tubes, floor, and interior fittings in all herds, as well as the top of the laser or camera and the premilking teat cleaning system in the AMS herds. Swab samples were also collected from water troughs in the milking department and the cubicle bases in the calving pens.

For dry sites, the swab was first dipped in the transport medium before it was rolled over the sample area. The samples were chilled immediately and transported to the laboratory in polystyrene boxes with cooling. In addition, hock lesion scoring was performed for at 
least 20 cows (or all cows if $<20$ cows in the herd), using score 0 (no lesion, or hairless patches $<2 \mathrm{~cm}$ in diameter), 1 (hairless patches $>2 \mathrm{~cm}$ in diameter, or scars $>3 \mathrm{~mm}$ ), or 2 (areas of skin damage $>2 \mathrm{~cm}$ in diameter).

\section{Sample Preparation and Analyses of Samples}

All milk samples were cultured upon arrival at the laboratory according to standard procedures (Hogan et al., 1999). Thereafter, $250 \mu \mathrm{L}$ from each quarter milk sample of each cow were mixed to a composite sample (cow sample), which was analyzed by quantitative (q) PCR, as described herein.

Samples from body sites and the environment were first screened by qPCR, and only qPCR-positive samples were cultured. The qPCR was performed upon arrival at the laboratory, and samples were thereafter frozen at $-20^{\circ} \mathrm{C}$ for up to 2 mo before culturing.

The qPCR detected the SDSD-specific genes rihC and 16S (Smistad et al., 2021). Briefly, DNA was extracted from $250 \mu \mathrm{L}$ of transport medium using MagNA Pure DNA and and NA LV Kit (Roche), and real-time PCR was performed using the Brilliant Multiplex qPCR Master Mix and an AriaMx instrument (both from Agilent).

Swab samples with a qPCR cycle quantity $\leq 40$ were cultured. The swabs in transport medium were thawed and plated on blood agar plates (Oxoid) with bovine blood supplemented with $0.5 \mathrm{~g} / \mathrm{L}$ esculin and Streptococcus supplement SR126 (Oxoid), with final concentrations of $2.5 \mathrm{mg} / \mathrm{L}$ oxolinic acid and $5 \mathrm{mg} / \mathrm{L}$ colistin sulfate. The plates were incubated anaerobically at $37^{\circ} \mathrm{C}$ for $24 \mathrm{~h}$. Putative SDSD colonies were identified with MALDI-TOF (Microflex LT System, Bruker Daltonics).

\section{Genome Sequencing of SDSD Isolates and Bioinformatics}

DNA Extraction and Sequencing. All isolates obtained from the 7 herd visits $(A-G)$, as well as 2 isolates collected in 2019 from milk samples from herds A and G, were sequenced. In addition, 20 isolates from quarter milk samples (from 20 different herds) were arbitrarily selected from a collection of SDSD isolates obtained during 2019 at the TINE Mastitis Laboratory. The isolates had been collected by freezing the first SDSD isolates obtained from clinical mastitis $(\mathrm{n}=5)$ and subclinical mastitis $(\mathrm{n}=5)$ every month between April and November 2019. Of the 90 sequenced isolates, 20 have been described previously (Porcellato et al., 2021). All genomes are available at DDBJ/ENA/GenBank under the BioProject PRJEB42928.
DNA was extracted using MagNA Pure DNA and the NA SV Kit (Roche), using an input of $200 \mu \mathrm{L}$, and the DNA Blood ds SV protocol optimized for doublestranded DNA and WGS. The template was eluted in $50 \mu \mathrm{L}$ of the kit elution buffer.

Genomic DNA was quantified using a Qubit 3.0 fluorometer (Life Technologies) normalized to $0.2 \mathrm{ng} / \mu \mathrm{L}$. The sequencing library was prepared using the Nextera XT DNA Sample Prep Kit (Illumina) before sequencing on the Illumina MiSeq platform (Illumina) and V3 chemistry.

Quality Control and Assembly of Sequences. Raw reads were quality controlled and assembled using the Ellipsis pipeline (Norwegian Veterinary Institute, 2021a). FastQC (Babraham Bioinformatics, 2019a) was used for read quality control, followed by trimming with Trim-Galore (Babraham Bioinformatics, 2019b). Genome assembly was performed by Unicycler (Wick et al., 2017) version 0.4.8 in normal mode. Genome assemblies were quality checked using QUAST (Gurevich et al., 2013). Assemblies were excluded if the total length was longer than $3 \mathrm{Mbp}$ or if the number of contigs exceeded 500. For multilocus sequence type (MLST) assignment, the draft genome was used as input in the MLST 2.0 (cge.cbs.dtu.dk/services/MLST) using the MLST scheme of Streptococcus dysgalactiae ssp. equisimilis (McMillan et al., 2010; Porcellato et al., 2021). To test whether any sequence type (ST) was associated with specific sampling sites (milk, body sites, environment), the frequency of each ST in each site was compared against that of other ST (as one group) using Fisher's exact test. Only those ST with at least 5 isolates were assessed. For isolates from milk, the association between ST and clinical manifestation (subclinical vs. clinical mastitis) was tested using Fisher's exact test.

Genome assemblies were checked for contaminant sequences using Kraken2 (Wood et al., 2019), version 2.0.9. Contaminant genomes were excluded if (1) the size of the contigs identified as contaminants exceeded $10 \%$ of the total genome size and (2) contigs classified as SDSD covered less than $80 \%$ of the total genome size. Average coverage was calculated for each genome assembly. Reads were mapped to their respective genome using BWA (Li and Durbin, 2009) version 0.7.17, and sorted using SAMtools (Li et al., 2009) version 1.9. BEDtools (Quinlan and Hall, 2010) version 2.27.1 was used to extract the coverage information from the BAM file, and the average nucleotide coverage in each genome was calculated. Genome assemblies with less than $30 \times$ average coverage were excluded from further analysis.

Phylogenetic Analysis. All genomes that passed quality control were included in the phylogenetic analy- 
sis, performed by the ALPPACA pipeline (Norwegian Veterinary Institute, 2021b). First, the genomes were annotated using Prokka (Seemann, 2014) version 1.14.5. Then, the pangenome was predicted and core gene alignment generated by Panaroo (Tonkin-Hill et al., 2020) version 1.2.2. The core gene alignment was de-duplicated with Seqkit (Shen et al., 2016) version 0.12 .0 and then used to reconstruct the phylogeny with IQ-Tree (Nguyen et al., 2015) version 1.6.12, using ultrafast bootstrap (Hoang et al., 2018) and model finder plus (Kalyaanamoorthy et al., 2017). The de-duplicated alignment was also used to calculate the pairwise SNP distances with snp-dists (https://github.com/ tseemann/snp-dists) version 0.6.3. The phylogenetic tree was visualized in $\mathrm{R}$ ( $\mathrm{R}$ Core Team, 2021) version 4.0.5, using the ggtree package (Yu et al., 2017, 2018; $\mathrm{Yu}, 2020)$ version 2.4.2.

Following inspection of the phylogenetic tree, subclades of interest were subjected to a phylogenetic analysis with a higher resolution within the ALPPACA pipeline. First, the core genome was predicted using ParSNP (Treangen et al., 2014) version 1.5.3. The alignment was de-duplicated as previously described, and recombinant areas were identified with Gubbins (Croucher et al., 2015) version 2.4.1. The recombinant areas were masked from the de-duplicated core gene alignment with Maskrc-svg (https://github.com/ kwongj/maskrc-svg) version 0.5. Then, IQ-Tree reconstructed the phylogeny from the masked alignment, and snp-dists calculated the pairwise SNP distances. The trees were visualized as described earlier.

The within-farm genetic diversity was assessed for farms with 5 or more isolates sequenced, and was based on the number of different ST per farm and the SNP distances between isolates from the same ST. To evaluate the genetic diversity of SDSD between the 27 farms, 1 isolate per ST per farm was selected for comparison.

\section{RESULTS}

\section{Descriptive Analyses and Risk Factor Study}

Of the 984 farms (215 cases and 769 controls) that fulfilled the inclusion criteria, 561 farmers $(57 \%)$ responded to the questionnaire. Twelve responses were excluded due to errors in herd identification, which made it impossible to match with the NHRS data. Responses from 549 farms, 132 case herds and 417 control herds, were available for descriptive analysis (Supplemental Table S1, https://doi.org/10.5281/zenodo.5959175). The median herd size was 30 cows (interquartile range $24-45$ cows), and the median yearly milk yield was 8,542 $\mathrm{kg}$ (interquartile range 7,858-9,105 kg). Norwegian Red was the main breed in 513 (94\%) of the included herds.
Therefore, breed was not included in the risk factor analysis. Freestall housing was associated with case herds $\left(\chi^{2} P=0.001\right)$, and the multivariable analyses included only the 365 freestall herds.

A total of 28 variables were screened in the univariable analyses, whereof 6 had a $P<0.2$ and were tested in the multivariable model (Supplemental Table S1). For the multivariable analyses, 6 observations were excluded due to missing values in the variables "cubicle base," "type of flooring," or both. The final data set used in the multivariable model included 359 herds: 102 case herds and 257 control herds. The variable "system for scraping/removing manure" was excluded due to collinearity with type of flooring.

The final logistic multivariable regression model identified milking parlor, closed floor in the alleys, and rubber mats as risk factors for being a case herd (Table 1). The interaction terms milking system $\times$ flooring type and milking system $\times$ herd size and flooring $\times$ herd size were tested but found not significant $(P>$ 0.05). The model showed good fit according to the Hosmer-Lemeshow goodness of fit test, with $\chi^{2}(8 \mathrm{df})$ $=7.18, P=0.52$.

\section{Sources and Genotypes of SDSD}

Herd characteristics of the 7 visited farms, the number of samples, and the proportions of SDSD-positive samples per farm are presented in Table 2. Farm G was organic, and the remaining 6 were conventional. Norwegian Red was the main breed on 6 of the farms. The seventh (farm A) had Norwegian Red and other breeds.

Milk samples from 74 cows were obtained (range 10-13 per farm). Of these, 13 cows had SDSD-IMI (range 0-4 per farm). Other cow-level bacterial findings were as follows: no growth $(\mathrm{n}=19)$, non-aureus staphylococci $(\mathrm{n}=18)$, Staphylococcus aureus $(\mathrm{n}=9)$, contamination $(\mathrm{n}=5)$, Streptococcus uberis $(\mathrm{n}=4)$, Streptococcus agalactiae $(\mathrm{n}=2)$, and other bacteria ( $\mathrm{n}$ $=4)$.

Results from qPCR analyses and bacterial culture of extramammary samples are described in Table 3 . The main SDSD-positive sampling sites by qPCR were wounds, teat or udder skin, and cubicle bases, whereas samples from mucous surfaces (nostrils, rectum, and vagina) and water troughs were the least frequently positive. Wound samples and samples from cubicle bases were most frequently culture positive.

\section{Genomic Analyses}

Quality Control and Contamination Screening. Quality control of altogether 90 sequenced SDSD isolates from 27 herds resulted in exclusion of 14 iso- 
lates from phylogenetic analyses due to contamination $(\mathrm{n}=10)$ and low coverage $(\mathrm{n}=4)$. However, it was possible to determine the ST for 13 of the excluded isolates. Hence, ST was determined for 89 isolates, and 76 isolates were included in the phylogenetic analyses.

Multilocus Sequence Typing. Eight different ST were identified among the 89 isolates for which ST could be determined. Five of these were allocated to isolates from more than one farm, including isolates from farm visits and from the mastitis laboratory (Table 4). Of these, ST453 predominated, compromising $43 \%$ of the isolates, found in 15 of the 27 herds. The remaining ST were detected in 4 herds (ST 301 and 302), 3 herds (ST 306 and 531), and 1 herd (ST 454, 532, and 524). From farms $\mathrm{A}$ and $\mathrm{G}$, sequenced isolates were collected both from the mastitis laboratory in 2019 and at farm visits in 2020. However, in both cases, the ST of the 2019 isolates were different from the 2020 isolates. Among isolates from the 7 farm visits in 2020, the numbers of ST per farm were 1 (farms A and F), 2 (farms B and D), or 3 (farm C and E). Farm G had only 1 available isolate.

Associations between sampling site (milk, body site, environment) and ST (ST 453, 302, 301, and 531 vs. all others) were tested but were found not significant (Fisher's exact $P>0.05$ ).

A total of 37 isolates from milk samples from 27 herds were sequenced. Of these, 10 isolates from 10 herds were from cows with clinical mastitis. These belonged to ST $453(\mathrm{n}=5), 306(\mathrm{n}=2), 301(\mathrm{n}=1), 531(\mathrm{n}=$ $1)$, and $532(\mathrm{n}=1)$. We found no significant association between ST and clinical manifestation (clinical vs. subclinical mastitis). Both ST 302 (farms C and E) and
453 (farm D) were found in IMI from more than one cow in the same herd.

Pangenome and Phylogenetic Analyses. The pangenome analysis identified 3,017 genes in the 76 genomes. Of these, 1,505 genes were categorized as core genes and were used to reconstruct the phylogeny. In the phylogenetic tree, the median SNP distance was 10,268 , with a range of 0 to 16,481 . The phylogenetic tree divided into 8 clades that corresponded with ST (Figure 1). The majority of isolates within the same ST clustered closely. Exceptions were observed for one ST 453 isolate, 3 ST 301, and 1 ST 306 (Figure 1). Four of the visited farms had one dominating ST ( $>80 \%$ of the isolates): farms A and D (ST 453), farm B (ST 301), and farm F (ST 302).

Separate phylogenetic analyses for ST 453, 302, and 531 , with the origin of the isolates (farm and type of sample) are presented in Figures 2, 3, and 4. For ST 301 , one subcluster included 8 isolates from body sites $(\mathrm{n}=7)$ and one from the environment of farm B. The alignment of the ST 453 core genome had an average genome coverage of $83.4 \%$, with a median SNP distance of 81, ranging from 1 to 223 SNPs. Within ST 453 , the median SNP distance between strains from different farms was 66 (range 14-168). For the ST 302 alignment, the core genome had an average coverage of $86.0 \%$, with a median SNP distance of 91 and a range of 1 to 114 SNPs. Finally, for the ST 531 alignment, the core genome average coverage was $95.1 \%$, with a median SNP distance of 79 and a range of 2 to 97 SNPs. Isolates from the same farm belonging to the same ST were more closely related (median core gene SNP distances between 2 and 18). There was one

Table 1. Herd variables associated with a high incidence of Streptococcus dysgalactiae ssp. dysgalactiae IMI (case herds) in a multivariable logistic regression model ${ }^{1}$

\begin{tabular}{|c|c|c|c|c|c|}
\hline Variable and category & $\mathrm{N}$ case herds $(\%)$ & $\mathrm{N}$ control herds $(\%)$ & $\mathrm{OR}^{2}$ & $95 \% \mathrm{CI}$ & $P$-value \\
\hline \multicolumn{6}{|l|}{ Milking system } \\
\hline $\mathrm{AMS}^{3}$ & $68(67)$ & $219(85)$ & Referent & & \\
\hline Parlor & $34(33)$ & $38(15)$ & 2.9 & $1.6-5.1$ & $<0.001$ \\
\hline \multicolumn{6}{|l|}{ Type of flooring ${ }^{4}$} \\
\hline Slatted & $62(61)$ & $204(79)$ & Referent & & \\
\hline Closed & $36(35)$ & $46(18)$ & 2.9 & $1.6-5.1$ & $<0.001$ \\
\hline Other & $4(4)$ & $7(3)$ & 1.8 & $0.49-6.7$ & 0.371 \\
\hline \multicolumn{6}{|l|}{ Cubicle bases } \\
\hline Mattresses & $81(79)$ & $171(67)$ & Referent & & \\
\hline Rubber mats & $21(21)$ & $86(33)$ & 2.5 & $1.4-4.4$ & 0.003 \\
\hline Herd size ${ }^{5}$ & & & 1.0 & $0.99-1.02$ & 0.616 \\
\hline \multicolumn{6}{|c|}{${ }^{1}$ Data from the Norwegian herd recording system and a farmer survey, Norway 2017-2018. Number of herds il } \\
\hline
\end{tabular}




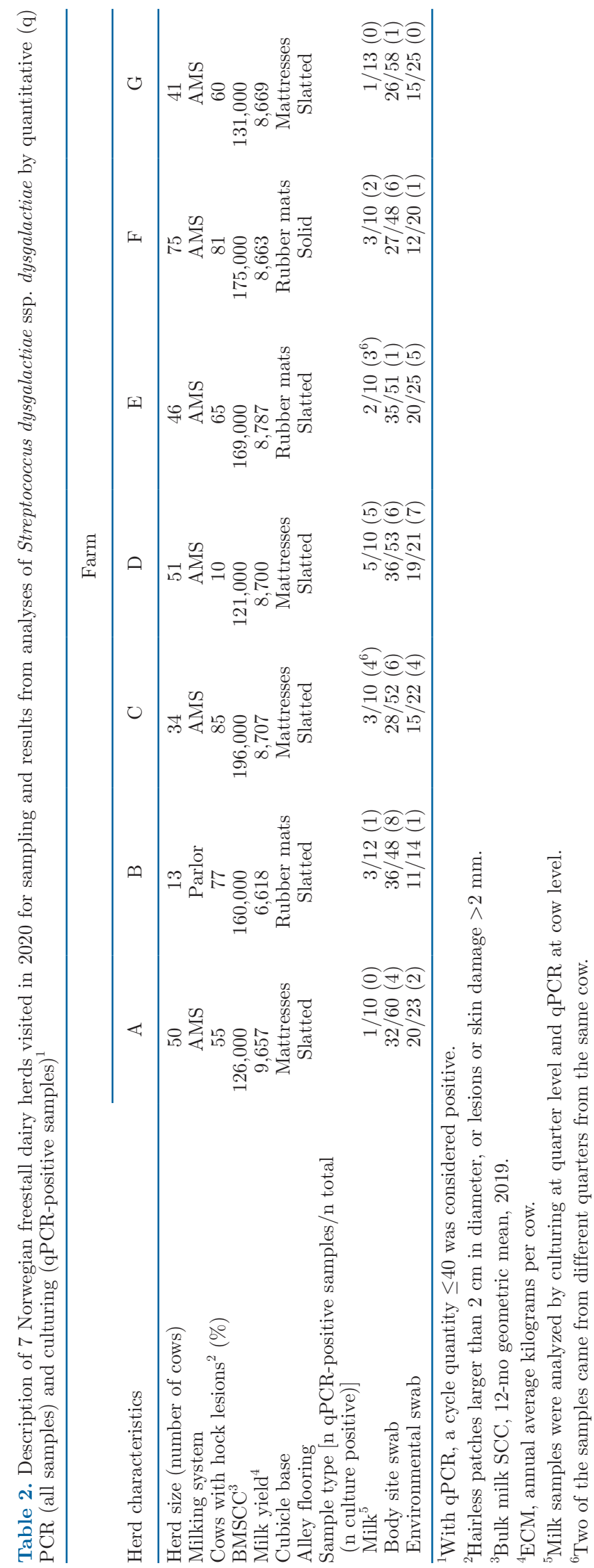

exception to this; the 10 ST 453 isolates from farm D divided into 2 subclusters with a maximum core gene SNP distance of 152 .

\section{DISCUSSION}

The current study adds some pieces to the puzzle of sources and potential transmission routes of SDSD in Norwegian bovine dairy herds. To our knowledge, it is the first study to systematically investigate risk factors for SDSD-IMI as well as bacterial sources in bovine dairy herds, and to use WGS to compare the betweenherd and within-herd genomic diversity of SDSD.

Freestall housing was associated with a high incidence of SDSD-IMI in the univariable analyses. This may explain why the prevalence of SDSD-IMI has increased in Norway in the last decades (TINE, 2020), because a shift toward freestall housing has occurred following a regulatory enforced transition from tiestalls (Norwegian Ministry of Agriculture and Food, 2004). Although freestall housing systems differ, they have in common that the cows move freely and come into direct and indirect contact with each other more frequently than in tiestalls. For example, the cubicles are shared between cows, creating potential contact surfaces for transmission. Furthermore, it is more feasible to practice a strict milking order according to infection status in tiestalls than in freestalls.

In the risk factor study, which included freestalls only, several risk factors were identified for case herds, that is, herds with a higher incidence of SDSD-IMI. Farms with closed floors in alleys had an increased risk of being a case herd compared with those with slatted floors. The mechanisms of this effect are not completely clear, but it could be related to a build-up of feces and slurry between floor scrapings in these systems, or to the higher humidity in barns with closed floors, in particular if drainage is poor.

In agreement with the findings of Taponen et al. (2017), our study identified parlor milking as a risk factor for being a case herd. Taponen et al. proposed that this could be associated with barn design, which may be a plausible explanation also in Norway. Most dairy barns built after 2010 in Norway are designed with slatted floors and have AMS, whereas closed flooring and milking parlors are more common in older freestall barns or barns rebuilt from tiestall barns. Another possible explanation for the association with the milking system may be differences in the cleaning of the teat cup liners, and hence the possibility for bacterial transfer during milking. The flushing or steaming of the teat cup liners between each cow in AMS herds is rarely practiced in parlor systems in Norway and may reduce 
Table 3. Frequency of detection of Streptococcus dysgalactiae ssp. dysgalactiae by quantitative (q) PCR (all samples) and culture (qPCRpositive samples) in 7 Norwegian freestall bovine dairy herds ${ }^{1}$

\begin{tabular}{|c|c|c|c|c|}
\hline Source & $\mathrm{N}$ & $\mathrm{N}$ qPCR positive (\%) & $\mathrm{N}$ culture positive $(\%)$ & Median cq 16S (range) \\
\hline Body site & 370 & $220(60)$ & $32(9)$ & $33(17.1-39.6)$ \\
\hline Udder skin & 71 & $59(83)$ & $5(7)$ & $34.1(28.0-39.0)$ \\
\hline Tonsil calf & 24 & $12(50)$ & $1(4)$ & $32.2(28.6-36.2)$ \\
\hline Vagina & 34 & $6(18)$ & $0(0)$ & $36.0(31.8-39.6)$ \\
\hline Environmental source & 150 & $112(75)$ & $21(14)$ & $33.2(26.8-39.1)$ \\
\hline Cubicle base & 55 & $52(95)$ & $12(22)$ & $33.0(26.8-39.1)$ \\
\hline Calving pen & 9 & $8(89)$ & $1(11)$ & $34.0(27.7-38.8)$ \\
\hline Milking machine ${ }^{3}$ & 60 & $35(58)$ & $6(10)$ & $33.1(29.6-37.3)$ \\
\hline Water trough & 13 & $5(38)$ & $1(8)$ & $36.0(30.6-37.4)$ \\
\hline Other environmental sample ${ }^{4}$ & 13 & $12(92)$ & $1(8)$ & $34.6(32.0-38.5)$ \\
\hline Total & 595 & $350(59)$ & $66(11)$ & $33.2(17.1-39.6)$ \\
\hline
\end{tabular}

${ }^{1}$ With qPCR, a cycle quantity (cq) $\leq 40$ was considered positive.

${ }^{2}$ Mainly mild hock lesions.

${ }^{3}$ Swabs were collected from liners, milk tubes, floor, and interior fittings of the milking system (all herds), and from the top of the laser or camera and the pre-milking teat cleaning system (automatic milking system herds).

${ }^{4}$ Floor, interior fittings, concentrate station.

the transmission of bacteria at milking in AMS herds (Skarbye et al., 2020).

Finally, herds with rubber mats as cubicle bases had a higher risk of being case herds compared with herds with mattresses. Rubber mats are generally more compact than mattresses and have been associated with hock lesions (Kielland et al., 2009; Ekman et al., 2018). A high proportion of wound samples $(92 \%)$ were qPCR positive in this study, and more than $20 \%$ of these were culture positive, often in almost pure growth (results not shown). Rubber mats may indirectly increase the incidence of SDSD-IMI by causing hock lesions where SDSD can multiply.

To further understand the underlying mechanisms of the association between barn type and the identified risk factors for a high incidence of SDSD-IMI in herds, we visited 7 freestall herds for bacteriological sampling. The main SDSD-positive sampling sites were wounds, teat or udder skin, and cubicle bases, whereas samples from mucous surfaces (nostrils, rectum, and vagina) were the least frequently positive. The main niche of SDSD appears to be the skin of the animals (Mundt, 1982), but the high positivity rate of environmental samples suggests that animals shed bacteria to the environment and that the environment plays a role in transmission of SDSD.

The most likely sources for contamination of the environment with SDSD is milk from cows with IMI, as well as the skin and wounds of the cows. The bacterial load in milk from cows suffering SDSD-IMI can be high (Hamel et al., 2021), and milk leakage from these cows is probably an important contribution to the environmental bacterial load. Fecal shedding may be less important as a source of SDSD to the environment because a relatively low proportion of rectal samples were qPCR positive (15\%), none of which were culture positive.

However, the barn environment is not necessarily a continuous reservoir of viable SDSD. In unpublished pilot studies, we were only able to retrieve SDSD by

Table 4. Distribution of Streptococcus dysgalactiae ssp. dysgalactiae multilocus sequence types in isolates from 27 Norwegian dairy herds; isolates were collected from farm visits $(\mathrm{n}=$ number of isolates, from 7 farms) and from routine analyses at the TINE Mastitis Laboratory, Molde, Norway ( $\mathrm{n}=$ number of isolates, from 20 farms across Norway)

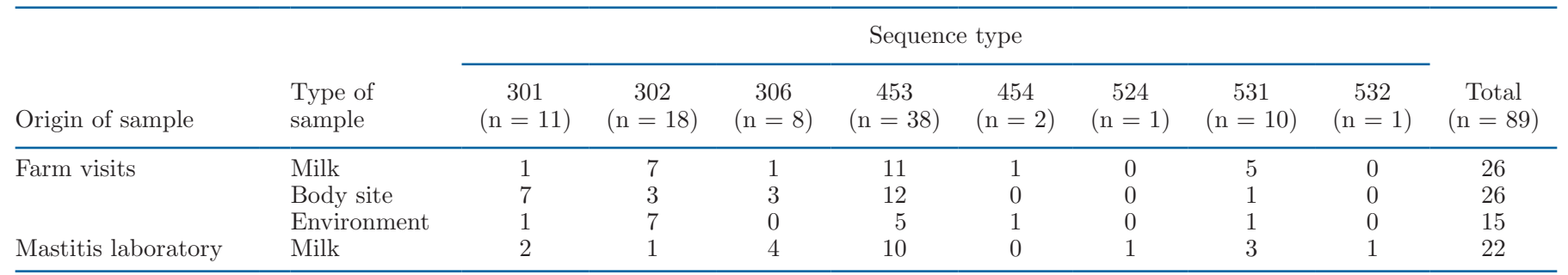




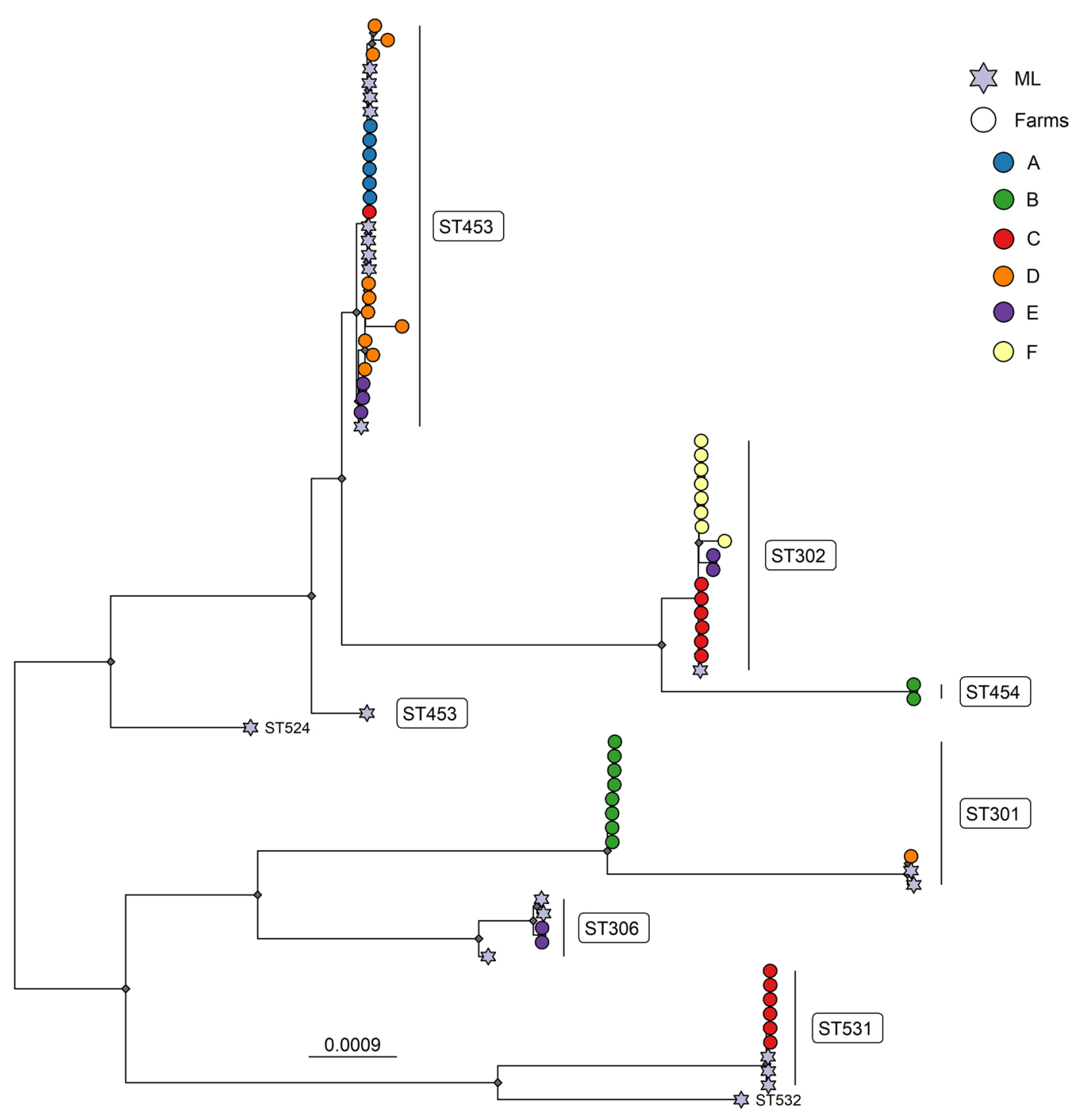

Figure 1. Maximum likelihood phylogenetic reconstruction based on an alignment of 1,505 core genes from 76 Streptococcus dysgalactiae ssp. dysgalactiae isolates. Nodes with high bootstrap support $(\geq 95)$ are denoted with a diamond. Tip shapes represent sample origin: star $=$ ML (mastitis laboratory), circle $=$ isolates from farm visits (farms A-F). Colors represent different farms and the mastitis laboratory $($ lilac). ST $=$ sequence type.

culturing for $1 \mathrm{~d}$ after spiking of manure taken from the floor of a bovine dairy herd and keeping it at $20^{\circ} \mathrm{C}$ under aerobic conditions. In contrast, we were able to culture Strep. agalactiae for almost 3 wk under equivalent conditions. Hence, although SDSD may be present in the environment and can transmit to cows from there, it is unlikely to multiply and survive for extended periods outside the cow. This would mean that the classical preventive measures against contagious udder pathogens, targeting infected udders, are relevant for SDSDIMI. We propose, however, that wound prevention and increased frequency of scraping and improved hygiene in cubicle bases should be included in any program to reduce SDSD-IMI in a herd.
Whole-genome sequencing found no associations between site of isolation and ST, which indicates that bacteria can transmit from animals to the environment and vice versa. It also showed that the same SDSD strain caused IMI in multiple cows in the same herd. Similar findings have been described by others (Lundberg et al., 2016; Wente and Krömker, 2020) and can be taken as evidence of direct or indirect transmission between animals. Further studies are needed to determine whether some strains of SDSD have greater potential for contagious spread than others.

Phylogenetic analysis revealed a clonal population structure, with relatively few sequence types detected in the investigated herds and a high similarity between 


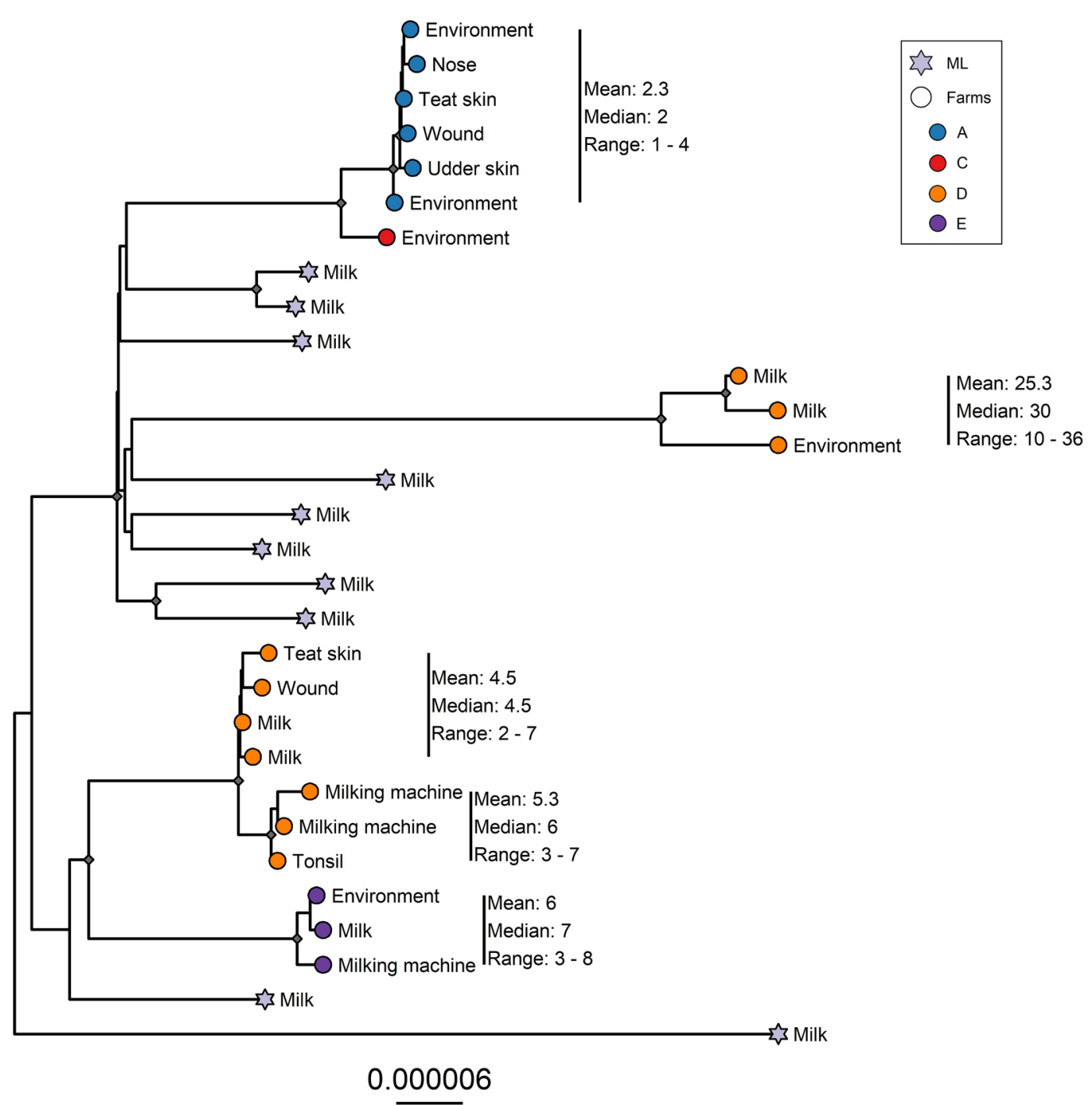

Figure 2. Maximum likelihood phylogenetic reconstruction based on the core genome alignment of the 30 Streptococcus dysgalactiae ssp. dysgalactiae sequence type 453 isolates. Nodes with high bootstrap support are denoted with a diamond. Tip shapes represent sample origin: star $=$ ML (mastitis laboratory), circle $=$ other (farm visits, farms A-E). Colors represent different farms and the mastitis laboratory (lilac). Tip labels represent sample type. SNP distance (number of SNPs) statistics (mean, median, range) are presented for each clade of interest.

isolates from the same ST. Furthermore, a modest number of ST were identified among the 89 sequenced SDSD isolates from 27 geographically spread farms, and one ST (ST453) was identified in 15 different herds. In a previous study, we sequenced SDSD from different species and found that SDSD delineated according to host species (Porcellato et al., 2021). By documenting that a few SDSD genotypes are more prevalent and can be found in geographically spread bovine dairy herds, our study supports the existence of bovine-adapted SDSD. Recent transmission between herds is an alternative explanation, but an epidemiological link between all these herds is unlikely. The greater SNP distance between isolates from different farms compared with isolates from the same farm also makes recent transmission between herds a less likely explanation.

An important question of this study was whether SDSD is a contagious udder pathogen or more of an opportunist. Traditionally, udder pathogens have been defined as "contagious" or "environmental," referring to the bacterial reservoirs and their principal modes of transmission (Schukken et al., 1991; Todhunter et al., 1995; Oliver et al., 2011). However, this binary classification is misleading for many important udder pathogens, including the streptococci (Klaas and Zadoks, 2018), and it may be more accurate to use the terms "environmental" or "cow-adapted" to describe the main reservoirs of the bacteria, and "opportunistic" 


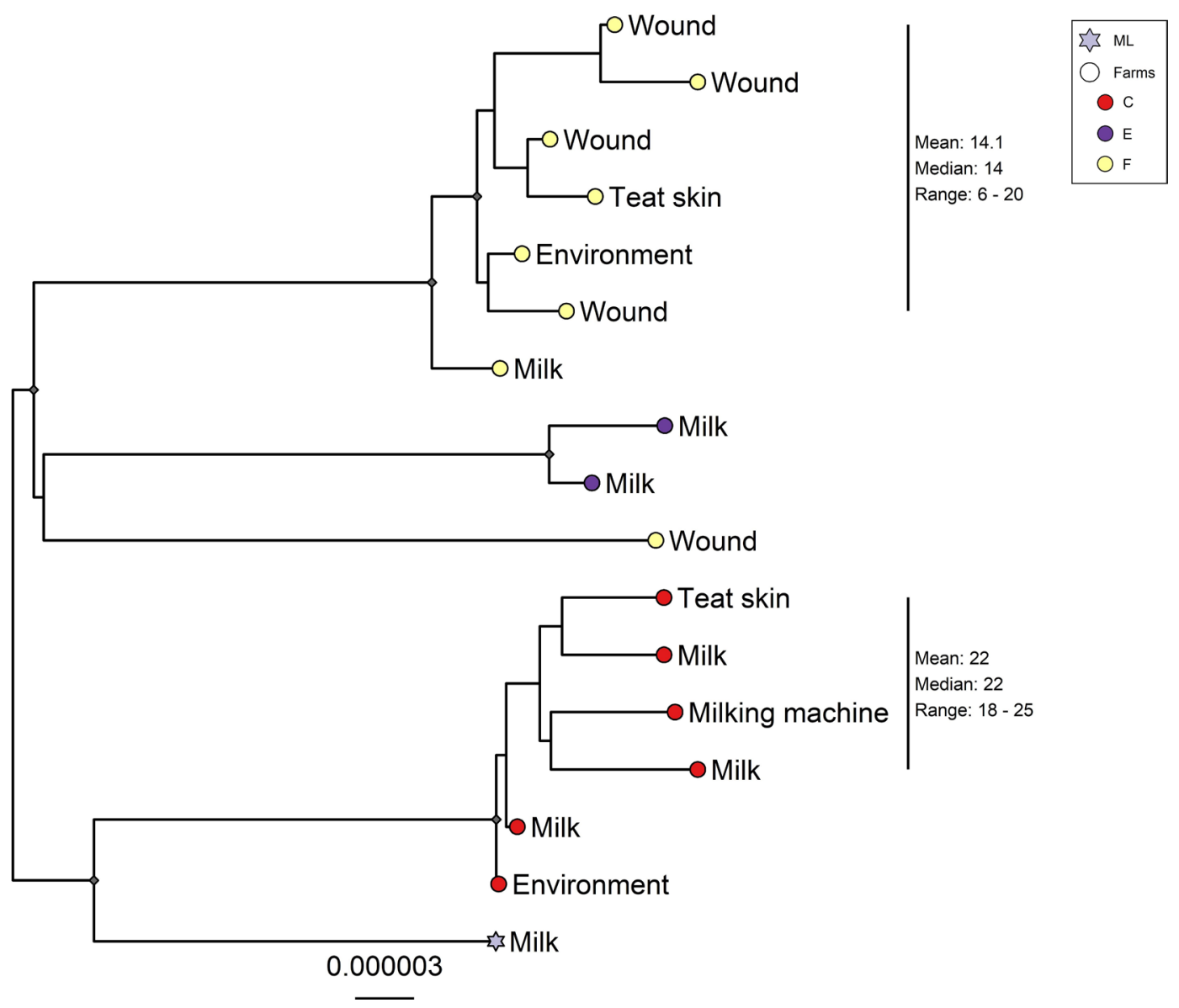

Figure 3. Maximum likelihood phylogenetic reconstruction based on the core genome alignment of the 17 Streptococcus dysgalactiae ssp. dysgalactiae sequence type 302 isolates. Nodes with high bootstrap support are denoted with a diamond. Tip shapes represent sample origin: star = ML (mastitis laboratory), circle = other (farm visits, farms C, E, and F). Colors represent different farms and the mastitis laboratory (lilac). Tip labels represent sample type. SNP distance (number of SNPs) statistics (mean, median, range) are presented for each clade of interest.

or "contagious" to describe their mode of transmission (Vanderhaeghen et al., 2015). Although our study does not fully answer these questions, it does suggest that the cow is the main niche of SDSD in dairy herds. Based on our findings, SDSD cannot unequivocally be categorized as opportunistic or contagious, but appears to be an opportunist that can also behave contagiously in certain situations

\section{CONCLUSIONS}

A high incidence of IMI caused by SDSD in Norwegian bovine dairy herds is associated with freestall housing. In addition to infected udders, sources of the bacterium include the skin and wounds of animals and the environment. This study suggests that SDSD is a cow-adapted udder pathogen with some potential for contagious spread and that the environment is one possible transmission route. The existence of SDSD on extramammary body sites is likely to contribute to the maintenance of bacterial reservoir in a herd after infected udders are treated. Therefore, wound prevention, appropriate hygiene, quality of cubicle bases, and control of IMI at dry-off are likely to be relevant measures to prevent SDSD-IMI in dairy herds.

\section{ACKNOWLEDGMENTS}

We thank the participating farmers and the team from TINE Mastitis Laboratory (Molde, Norway). Tormod Mørk (Norwegian Veterinary Institute, Ås, Norway), Anne Guro Larsgard (Geno SA, Hamar, Norway), Olav Østerås (TINE SA, Ås), and Basma Asal (Norwegian Veterinary Institute, Ås) are acknowledged for their valuable contributions. This research was conducted as a part of the project "Increasing sustainability of Norwegian food production by tackling streptococcal infections in modern livestock systems." The work re- 


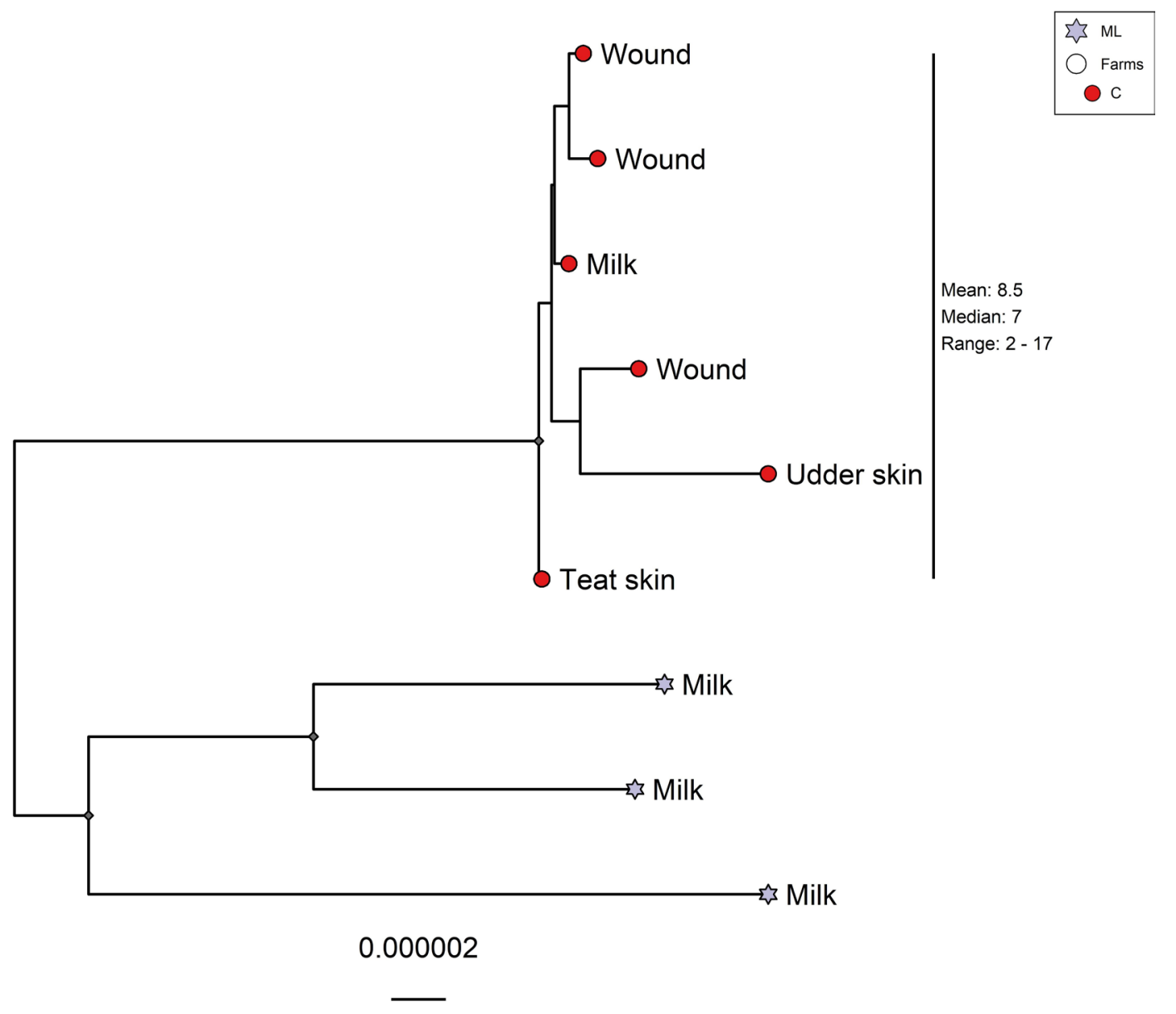

Figure 4. Maximum likelihood phylogenetic reconstruction based on the core genome alignment of the 9 Streptococcus dysgalactiae ssp. dysgalactiae sequence type 531 isolates. Nodes with high bootstrap support are denoted with a diamond. Tip shapes represent sample origin: star $=\mathrm{ML}$ (mastitis laboratory), circle $=$ other (farm visits, farm C). Colors represent different farms and the mastitis laboratory (lilac). Tip labels represent sample type. SNP distance (number of SNPs) statistics (mean, median, range) are presented for each clade of interest.

ceived financial contributions from the Norwegian Agricultural Agreement Research Fund (Oslo, Norway) and the Norwegian Research council (Oslo; grant numbers 280364 and 288917), the Norwegian Meat and Poultry Research Centre (Oslo), and TINE SA (Ås). The authors have not stated any conflicts of interest.

\section{REFERENCES}

Aarestrup, F. M., and N. E. Jensen. 1996. Genotypic and phenotypic diversity of Streptococcus dysgalactiae strains isolated from clinical and subclinical cases of bovine mastitis. Vet. Microbiol. 53:315323. https://doi.org/10.1016/S0378-1135(96)01221-7.

Babraham Bioinformatics. 2019a. FastQC. Accessed Jul. 1, 2021. https://www.bioinformatics.babraham.ac.uk/projects/fastqc/.

Babraham Bioinformatics. 2019b. Trim Galore. Accessed Jul. 1, 2021. https://www.bioinformatics.babraham.ac.uk/projects/trim galore/.

Barkema, H. W., Y. H. Schukken, T. J. G. M. Lam, M. L. Beiboer, G. Benedictus, and A. Brand. 1999. Management practices associated with the incidence rate of clinical mastitis. J. Dairy Sci. 82:16431654. https://doi.org/10.3168/jds.S0022-0302(99)75393-2.
Baseggio, N., P. D. Mansell, J. W. Browning, and G. F. Browning. 1997. Strain differentiation of isolates of streptococci from bovine mastitis by pulsed-field gel electrophoresis. Mol. Cell. Probes 11:349-354. https://doi.org/10.1006/mcpr.1997.0126.

Bramley, A. J., J. E. Hillerton, T. M. Higgs, and E. M. Hogben. 1985. The carriage of summer mastitis pathogens by muscid flies. Br. Vet. J. 141:618-627. https://doi.org/10.1016/0007-1935(85)90009 -0 .

Chirico, J., P. Jonsson, S. Kjellberg, and G. Thomas. 1997. Summer mastitis experimentally induced by Hydrotaea irritans exposed to bacteria. Med. Vet. Entomol. 11:187-192. https://doi.org/10 $.1111 /$ j.1365-2915.1997.tb00312.x.

Croucher, N. J., A. J. Page, T. R. Connor, A. J. Delaney, J. A. Keane, S. D. Bentley, J. Parkhill, and S. R. Harris. 2015. Rapid phylogenetic analysis of large samples of recombinant bacterial whole genome sequences using Gubbins. Nucleic Acids Res. 43:e15. https: //doi.org/10.1093/nar/gku1196.

Cruz Colque, J. I., L. A. Devriese, and F. Haesebrouck. 1993. Streptococci and enterococci associated with tonsils of cattle. Lett. Appl. Microbiol. 16:72-74. https://doi.org/10.1111/j.1472-765X.1993 .tb00346.x.

Daleel, E. E., and A. J. Frost. 1967. Some observations on the bacterial flora of the bovine tonsil. Br. Vet. J. 123:232-236. https://doi .org/10.1016/S0007-1935(17)39953-0. 
Dohoo, I., W. Martin, and H. Stryhn. 2014. Veterinary Epidemiologic Research. 2nd ed. VER Inc.

Duse, A., K. Persson-Waller, and K. Pedersen. 2021. Microbial aetiology, antibiotic susceptibility and pathogen-specific risk factors for udder pathogens from clinical mastitis in dairy cows. Animals (Basel) 11:2113. https://doi.org/10.3390/ani11072113.

Ekman, L., A.-K. Nyman, H. Landin, and K. Persson Waller. 2018. Hock lesions in dairy cows in freestall herds: A cross-sectional study of prevalence and risk factors. Acta Vet. Scand. 60:47. https: //doi.org/10.1186/s13028-018-0401-9.

Ericsson Unnerstad, H., A. Lindberg, K. Persson Waller, T. Ekman, K. Artursson, M. Nilsson-Öst, and B. Bengtsson. 2009. Microbial aetiology of acute clinical mastitis and agent-specific risk factors. Vet. Microbiol. 137:90-97. https://doi.org/10.1016/j.vetmic.2008 .12 .005 .

Gillespie, B., B. Jayarao, J. Pankey, and S. Oliver. 1998. Subtyping of Streptococcus dysgalactiae and Streptococcus uberis isolated from bovine mammary secretions by DNA fingerprinting. J. Vet. Med. B 45:585-593.

Gurevich, A., V. Saveliev, N. Vyahhi, and G. Tesler. 2013. QUAST: Quality assessment tool for genome assemblies. Bioinformatics 29:1072-1075. https://doi.org/10.1093/bioinformatics/btt086.

Hamel, J., Y. Zhang, N. Wente, and V. Krömker. 2021. Heat stress and cow factors affect bacteria shedding pattern from naturally infected mammary gland quarters in dairy cattle. J. Dairy Sci. 104:786-794. https://doi.org/10.3168/jds.2020-19091.

Heikkilä, A.-M., E. Liski, S. Pyörälä, and S. Taponen. 2018. Pathogen-specific production losses in bovine mastitis. J. Dairy Sci. 101:9493-9504. https://doi.org/10.3168/jds.2018-14824.

Hoang, D. T., O. Chernomor, A. Von Haeseler, B. Q. Minh, and L. S. Vinh. 2018. UFBoot2: Improving the ultrafast bootstrap approximation. Mol. Biol. Evol. 35:518-522. https://doi.org/10.1093/ molbev/msx 281

Hogan, J., R. Gonzalez, R. Harmon, S. Nickerson, S. Oliver, J. Pankey, and K. L. Smith. 1999. Laboratory Handbook on Bovine Mastitis. National Mastitis Council.

Kabelitz, T., E. Aubry, K. van Vorst, T. Amon, and M. Fulde. 2021. The role of Streptococcus spp. in bovine mastitis. Microorganisms 9:1497. https://doi.org/10.3390/microorganisms9071497.

Kalyaanamoorthy, S., B. Q. Minh, T. K. F. Wong, A. von Haeseler, and L. S. Jermiin. 2017. ModelFinder: Fast model selection for accurate phylogenetic estimates. Nat. Methods 14:587-589. https:// doi.org/10.1038/nmeth.4285.

Kielland, C., L. Ruud, A. Zanella, and O. Østerås. 2009. Prevalence and risk factors for skin lesions on legs of dairy cattle housed in freestalls in Norway. J. Dairy Sci. 92:5487-5496. https://doi.org/ 10.3168/jds.2009-2293.

Klaas, I. C., and R. N. Zadoks. 2018. An update on environmental mastitis: Challenging perceptions. Transbound. Emerg. Dis. 65:166-185. https://doi.org/10.1111/tbed.12704.

Li, H., and R. Durbin. 2009. Fast and accurate short read alignment with Burrows-Wheeler transform. Bioinformatics 25:1754-1760. https://doi.org/10.1093/bioinformatics/btp324.

Li, H., B. Handsaker, A. Wysoker, T. Fennell, J. Ruan, N. Homer, G. Marth, G. Abecasis, R. Durbin, and 1000 Genome Project Data Processing Subgroup. 2009. The Sequence Alignment/Map format and SAMtools. Bioinformatics 25:2078-2079. https://doi.org/10 .1093/bioinformatics/btp352.

Lundberg, A. 2015. Mastitis in Dairy Cows: Genotypes, Spread, and Infection Outcome of Three Important Udder Pathogens. Faculty of Veterinary Medicine and Animal Science, Department of Clinical Sciences, and National Veterinary Institute, Department of Animal Health and Antimicrobial Strategies. Doctoral Thesis. Swedish University of Agricultural Sciences, Uppsala.

Lundberg, Å., A. Nyman, A. Aspán, S. Börjesson, H. E. Unnerstad, and K. P. Waller. 2016. Udder infections with Staphylococcus aureus, Streptococcus dysgalactiae, and Streptococcus uberis at calving in dairy herds with suboptimal udder health. J. Dairy Sci. 99:2102-2117. https://doi.org/10.3168/jds.2015-9487.

Lundberg, Å., A. Nyman, H. E. Unnerstad, and K. P. Waller. 2014. Prevalence of bacterial genotypes and outcome of bovine clinical mastitis due to Streptococcus dysgalactiae and Streptococcus uberis Acta Vet. Scand. 56:80. https://doi.org/10.1186/s13028-014-0080 -0 .

McMillan, D. J., D. E. Bessen, M. Pinho, C. Ford, G. S. Hall, J. MeloCristino, and M. Ramirez. 2010. Population genetics of Streptococcus dysgalactiae subspecies equisimilis reveals widely dispersed clones and extensive recombination. PLoS One 5:e11741. https:// doi.org/10.1371/journal.pone.0011741.

Mundt, J. O. 1982. The ecology of the streptococci. Microb. Ecol. 8:355-369. https://doi.org/10.1007/BF02010675.

Nguyen, L.-T., H. A. Schmidt, A. Von Haeseler, and B. Q. Minh. 2015. IQ-TREE: A fast and effective stochastic algorithm for estimating maximum-likelihood phylogenies. Mol. Biol. Evol. 32:268-274. https://doi.org/10.1093/molbev/msu300.

Norwegian Ministry of Agriculture and Food. 2004. FOR-2004-04-22665. Regulation on keeping cattle (in Norwegian). Accessed Dec. 21, 2021. https://lovdata.no/dokument/SF/forskrift/2004-04-22 -665 .

Norwegian Veterinary Institute. 2021a. Ellipsis. https://doi.org/10 .5281 /zenodo. 4563897 .

Norwegian Veterinary Institute. 2021b. ALPPACA. https://doi.org/10 .5281 /zenodo.4452122.

Oliver, S. P., G. M. Pighetti, and R. A. Almeida. 2011. Mastitis pathogens: Environmental pathogens. Pages 415-421 in Encyclopedia of Dairy Sciences, 2nd ed. J. W. Fuquay, ed. Academic Press.

Persson Waller, K., B. Bengtsson, A. Lindberg, A. Nyman, and H. Ericsson Unnerstad. 2009. Incidence of mastitis and bacterial findings at clinical mastitis in Swedish primiparous cows - Influence of breed and stage of lactation. Vet. Microbiol. 134:89-94. https:// doi.org/10.1016/j.vetmic.2008.09.004.

Porcellato, D., M. Smistad, S. B. Skeie, H. J. Jørgensen, L. Austbø, and O. Oppegaard. 2021. Whole genome sequencing reveals possible host species adaptation of Streptococcus dysgalactiae. Sci. Rep. 11:17350. https://doi.org/10.1038/s41598-021-96710-z.

Quinlan, A. R., and I. M. Hall. 2010. BEDTools: A flexible suite of utilities for comparing genomic features. Bioinformatics 26:841842. https://doi.org/10.1093/bioinformatics/btq033.

R Core Team. 2021. R: A Language and Environment for Statistical Computing. R Foundation for Statistical Computing. https://www .R-project.org/.

Schukken, Y. H., F. J. Grommers, D. Van de Geer, H. N. Erb, and A. Brand. 1991. Risk factors for clinical mastitis in herds with a low bulk milk somatic cell count. 2. Risk factors for Escherichia coli and Staphylococcus aureus. J. Dairy Sci. 74:826-832. https://doi .org/10.3168/jds.S0022-0302(91)78231-3.

Seemann, T. 2014. Prokka: Rapid prokaryotic genome annotation. Bioinformatics 30:2068-2069. https://doi.org/10.1093/ bioinformatics/btu153.

Shen, W., S. Le, Y. Li, and F. Hu. 2016. SeqKit: A cross-platform and ultrafast toolkit for FASTA/Q file manipulation. PLoS One 11:e0163962. https://doi.org/10.1371/journal.pone.0163962.

Skarbye, A. P., P. T. Thomsen, M. A. Krogh, L. Svennesen, and S. Østergaard. 2020. Effect of automatic cluster flushing on the concentration of Staphylococcus aureus in teat cup liners. J. Dairy Sci. 103:5431-5439. https://doi.org/10.3168/jds.2019-17785.

Smistad, M., T. S. Tollersrud, L. Austbø, D. Porcellato, C. Wolff, B. Asal, C. J. Phythian, O. Oppegaard, and H. J. Jørgensen. 2021. Molecular detection and genotype characterization of Streptococcus dysgalactiae from sheep flocks with outbreaks of infectious arthritis. Vet. Microbiol. 262:109221. https://doi.org/10.1016/j vetmic.2021.109221.

Statistics Norway. 2020. Statistics for Agriculture, Forestry, Hunting and Fishing. Accessed Aug. 31, 2021. https://www.ssb.no/en/jord -skog-jakt-og-fiskeri/statistikker/jordhus.

Taponen, S., E. Liski, A.-M. Heikkilä, and S. Pyörälä. 2017. Factors associated with intramammary infection in dairy cows caused by coagulase-negative staphylococci, Staphylococcus aureus, Streptococcus uberis, Streptococcus dysgalactiae, Corynebacterium bovis, or Escherichia coli. J. Dairy Sci. 100:493-503. https://doi.org/10 .3168/jds.2016-11465. 
TINE. 2020. Årsrapport Kukontrollen og Geitekontrollen [Results from the Norwegian Herd Recording System and Norwegian Goat Recording System]. Accessed Aug. 1, 2021. https://medlem .tine.no/fag-og-forskning/statistikk-2020-for-kukontrollen-og -geitekontrollen/_/attachment/inline/59a4610f-2d7e-4b6f-a37a -7f43c9977306:53e8e6153c6fa6348276b5cc0bc8e9b3a58d6079/ Statistikksamling\%20husdyrkontrollen\%202020.pdf.

Todhunter, D. A., K. L. Smith, and J. S. Hogan. 1995. Environmental streptococcal intramammary infections of the bovine mammary gland. J. Dairy Sci. 78:2366-2374. https://doi.org/10.3168/jds S0022-0302(95) 76864-3.

Tonkin-Hill, G., N. MacAlasdair, C. Ruis, A. Weimann, G. Horesh, J. A. Lees, R. A. Gladstone, S. Lo, C. Beaudoin, R. A. Floto, S. D. W. Frost, J. Corander, S. D. Bentley, and J. Parkhill. 2020. Producing polished prokaryotic pangenomes with the Panaroo pipeline. Genome Biol. 21:180. https://doi.org/10.1186/s13059 -020-02090-4.

Treangen, T. J., B. D. Ondov, S. Koren, and A. M. Phillippy. 2014. The Harvest suite for rapid core-genome alignment and visualization of thousands of intraspecific microbial genomes. Genome Biol. 15:524. https://doi.org/10.1186/s13059-014-0524-x.

Vanderhaeghen, W., S. Piepers, F. Leroy, E. Van Coillie, F. Haesebrouck, and S. De Vliegher. 2015. Identification, typing, ecology and epidemiology of coagulase negative staphylococci associated with ruminants. Vet. J. 203:44-51. https://doi.org/10.1016/j.tvjl 2014.11.001

Wang, S. M., M. A. Deighton, J. A. Capstick, and N. Gerraty. 1999. Epidemiological typing of bovine streptococci by pulsed-field gel electrophoresis. Epidemiol. Infect. 123:317-324. https://doi.org/10 .1017/S0950268899002745.

Wente, N., and V. Krömker. 2020. Streptococcus dysqalactiae - Contagious or environmental? Animals (Basel) 10:2185. https://doi.org/ 10.3390/ani10112185
Whist, A. C., O. Østerås, and L. Sølverød. 2007. Streptococcus dysgalactiae isolates at calving and lactation performance within the same lactation. J. Dairy Sci. 90:766-778. https://doi.org/10.3168/ jds.S0022-0302(07)71561-8.

Wick, R. R., L. M. Judd, C. L. Gorrie, and K. E. Holt. 2017. Unicycler: Resolving bacterial genome assemblies from short and long sequencing reads. PLOS Comput. Biol. 13:e1005595. https://doi .org/10.1371/journal.pcbi.1005595.

Wood, D. E., J. Lu, and B. Langmead. 2019. Improved metagenomic analysis with Kraken 2. Genome Biol. 20:257. https://doi.org/10 $.1186 / \mathrm{s} 13059-019-1891-0$.

$\mathrm{Yu}, \mathrm{G} .2020$. Using ggtree to visualize data on tree-like structures. Curr. Protoc. Bioinformatics 69:e96. https://doi.org/10.1002/cpbi .96

Yu, G., T. T.-Y. Lam, H. Zhu, and Y. Guan. 2018. Two methods for mapping and visualizing associated data on phylogeny using ggtree. Mol. Biol. Evol. 35:3041-3043. https://doi.org/10.1093/ molbev/msy194.

Yu, G., D. K. Smith, H. Zhu, Y. Guan, and T. T. Y. Lam. 2017. ggtree: An $\mathrm{R}$ package for visualization and annotation of phylogenetic trees with their covariates and other associated data. Methods Ecol. Evol. 8:28-36. https://doi.org/10.1111/2041-210X.12628.

\section{ORCIDS}

M. Smistad ๑ https://orcid.org/0000-0002-9282-3594

H. Kaspersen (1) https://orcid.org/0000-0002-9559-1303

C. Wolff $\odot$ https://orcid.org/0000-0002-8661-901X

D. Porcellato ( https://orcid.org/0000-0002-7460-534X

H. J. Jørgensen (® https://orcid.org/0000-0002-1788-9219 